\title{
Prognostic Impact of Semantic MRI Features on Survival Outcomes in Medulloblastoma: Does It Reflect or Transcend Radiogenomic Correlation?
}

\section{Archya Dasgupta}

Tata Memorial Centre

Tejpal Gupta ( $\nabla$ tejpalgupta@rediffmail.com )

Tata Memorial Hospital https://orcid.org/0000-0001-8256-9206

Madan Maitre

Tata Memorial Centre

Babusha Kalra

Tata Memorial Centre

Abhishek Chatterjee

Tata Memorial Centre

Rahul Krishnatry

Tata Memorial Centre

Jayant Sastri Goda

Tata Memorial Centre

Neelam Shirsat

Tata Medical Center

\section{Sridhar Epari}

Tata Memorial Centre

\section{Ayushi Sahay}

Tata Memorial Centre

\section{Amit Janu}

Tata Memorial Centre

\section{Sona Pungavkar}

Tata Memorial Centre

\section{Girish Chinnaswamy}

Tata Memorial Centre

Vijay Patil

Tata Memorial Centre

\section{Aliasgar Moiyadi}

Tata Memorial Centre

Prakash Shetty

Tata Memorial Centre

\section{Rakesh Jalali}

Tata Memorial Centre 


\section{Research Article}

Keywords: Magnetic resonance imaging (MRI), Medulloblastoma, Molecular Subgroups, Relapse, Radiogenomics, Survival

Posted Date: August 10th, 2021

DOl: https://doi.org/10.21203/rs.3.rs-790721/v1

License: 두 (i) This work is licensed under a Creative Commons Attribution 4.0 International License. Read Full License 


\section{Abstract \\ Background}

Imaging features are known to reflect inherent disease biology in various cancers including brain tumors. We report prognostic impact of magnetic resonance imaging (MRI) features on survival in patients with medulloblastoma treated between 2007 and 2018 at our institute.

\section{Methods}

Sixteen semantic imaging features (with pre-defined categories) were extracted from pre- and post-contrast T1weighted and T2-weighted MRI by consensus. Univariate analysis and multivariate Cox regression analysis were performed to assess correlation of semantic features with relapse-free survival (RFS) and overall survival (OS).

\section{Results}

The study cohort comprised of 171 medulloblastoma patients (median age 9 years) treated with maximal safe resection followed by risk-stratified adjuvant radio(chemo)therapy. A total of 55 patients experienced recurrent/progressive disease (commonly neuraxial metastases) resulting in 44 deaths including one treatmentrelated death. At a median follow-up of 45 months (inter-quartile range 19-65 months), 5-year Kaplan-Meier estimates of RFS and OS were $64 \%$ and $71 \%$, respectively. Semantic MRI features such as non-central tumor location on vertical axis, absence of brainstem involvement, $\leq 80 \%$ solid tumor area with contrast-uptake, heterogenous pattern of contrast-enhancement, necrosis, calcification, and T2-weighted heterogeneity were associated with significantly worse RFS and/or OS on univariate analysis. Cox regression analysis identified tumor location on the vertical axis, brainstem involvement, and calcification as independent prognostic factors impacting outcomes. Distinctive MRI features correlated with survival even within individual molecular subgroups of medulloblastoma.

\section{Conclusion}

Distinctive semantic MRI features correlate significantly with survival outcomes in medulloblastoma including within individual molecular subgroups reflecting their prognostic impact that transcends radiogenomic correlation.

\section{Introduction}

Medulloblastoma is the most common primary malignant brain tumor in the pediatric age group comprising 20$25 \%$ of all such neoplasms, but affects the adult age group (>18 years) much less frequently [1, 2]. Since its initial description as 'small blue round cell tumor' and identification as a 'single histological entity' nearly a century ago, we have come a long way in recognizing the inherent biological and clinical heterogeneity of medulloblastoma due to tremendous advances in genetics and molecular pathology in the last two decades [3-5]. Novel biological insights have led to consensus classification of medulloblastoma into 4 unique molecular subgroups - wingless (WNT), sonic hedgehog (SHH), Group 3, and Group 4, respectively [6], each with different developmental origins, 
distinct phenotypes, unique transcriptional profile, and variable clinical behavior and outcomes. This new histomolecular classification has now been incorporated in the updated World Heath Organization (WHO) classification of embryonal CNS tumors [7] and has led to the refinement of contemporary risk-stratification in the molecular era [8].

Traditionally, imaging has been used for diagnosis (characterization of lesion) and staging (assessment of disease extent) in oncology. However, in recent times, we have increasingly recognized that imaging features reflect underlying inherent disease biology $[9,10]$ including the dynamics of complex biological processes such as gene expression, proliferation, metabolism, and angiogenesis [11]. It has now become possible to extract meaningful information from routine images that go well beyond the diagnostic and staging roles of radiology to serve as independent predictive and prognostic biomarkers. Radiomics and radiogenomics [9-11] have emerged

as exciting avenues of research that aim to define relationships between non-invasive imaging features (radiophenotypes) and outcomes (survival, toxicity) or genomic data/molecular markers (molecular phenotypes) respectively. Magnetic resonance imaging (MRI) is the preferred first-line imaging modality in the diagnosis, staging evaluation, treatment planning, response assessment, and surveillance of brain tumors including medulloblastoma. In our previous study [12], we have demonstrated the utility of semantic MRI features extracted from pre-operative images to predict specific molecular subgroups in medulloblastoma with the best diagnostic performance for SHH and Group 4 medulloblastoma. Similar prediction accuracy was later reported using radiomics-based approaches with support machine vector classifier [13] and random forest model [14] to identify molecular subgroups. Several other studies have used semantic, radiomic, or spectroscopic features to predict molecular subgroups with acceptable accuracy $[15,16]$, but none of them have attempted to correlate specific imaging features with survival in medulloblastoma. In the present study, we report the prognostic impact of semantic MRI features on recurrence and survival in our institutional cohort of medulloblastoma across molecular subgroups as well as within individual molecular subgroups.

\section{Materials And Methods}

\section{Patient selection}

All patients with a histopathological diagnosis of medulloblastoma registered at our institute between 2007 to 2018 were screened for inclusion in the study. Only patients with available pre-operative MRI scans and adequate tissue material (fresh-frozen tumor tissues or formalin-fixed paraffin-embedded blocks) for molecular subgrouping were considered eligible for the present analysis. Patient, disease, treatment characteristics, and disease-related outcomes were extracted from hospital case files and electronic medical records supported by data from prospectively maintained neuro-oncology database as appropriate. This was a combined retrospective and prospective study with written informed consent and assent (as appropriate) obtained from patients and/or caregivers in the prospective cohort while waiver of consent was granted by our Institutional Ethics Committee for the retrospective cohort.

\section{Semantic MRI features}

Radiological characterization of tumors was done on the available pre-operative MRI in a multi-disciplinary neurooncology joint clinic comprising of faculty from various disciplines (neuro-radiology, neuro-surgery, radiation oncology, pediatric oncology, and neuro-pathology). Sixteen semantic MRI features (including pre-defined subcategories) such as tumor location, relationship with brainstem, maximum tumor size, contrast-enhancement 
pattern, T2-weighted characteristics, diffusion characteristics, tumor margin, peri-tumoral edema, intra-tumoral hemorrhage, necrosis, calcification, associated cysts, and hydrocephalus were extracted from pre- and postcontrast T1-weighted and T2-weighted images by consensus as described in detail previously [12].

\section{Molecular profiling}

Molecular subgroup affiliation (WNT, SHH, Group 3, and Group 4) was done on formalin-fixed paraffin-embedded blocks or fresh-frozen tumor tissues based on the differential expression of 12-protein coding genes and 9microRNAs using real-time reverse transcriptase polymerase chain reaction (RT-PCR) assay [17]. Tumors with poor tissue-preservation precluding definitive subgroup assignment and/or overlapping molecular features (nonWNT/nonSHH medulloblastoma - cannot classify further) were excluded from the analysis.

\section{Treatment details}

All patients underwent maximal safe resection followed by post-operative adjuvant radiotherapy (RT) with or without chemotherapy based on traditional clinico-radiological risk-stratification system [18]. Children with average-risk disease (defined as age $>3$ years, residual tumor $\leq 1.5 \mathrm{~cm}^{2}$, and no leptomeningeal metastases on neuraxial staging) were treated with reduced-dose craniospinal irradiation (CSI) to a dose of 23.4Gy/14 fractions plus posterior fossa/tumor-bed boost RT to a dose of $30.6 \mathrm{~Gy} / 17$ fractions for total primary-site dose of $54 \mathrm{~Gy} / 31$ fractions weeks followed by 6-cycles of adjuvant systemic chemotherapy starting at about 4 weeks from the end of RT after complete myelo-recovery. Adults ( $\geq 18$ years) with average-risk medulloblastoma were treated with full-dose CSI (35Gy/21 fractions) plus posterior fossa/tumor-bed boost RT (19.8Gy/11 fractions) for total primary-site dose of $54.8 \mathrm{~Gy} / 32$ fractions without adjuvant systemic chemotherapy. Patients with high-risk disease (defined as residual tumor $>1.5 \mathrm{~cm}^{2}$, presence of leptomeningeal metastases, or large-cell/anaplastic histology) were treated with full-dose (35Gy/21 fractions) or sometimes even extended-dose CSI (40Gy/24 fractions) with concurrent carboplatin plus posterior fossa/tumor-bed boost RT (14.4-19.8Gy) for total primarysite dose of 54-55Gy/32 fractions followed by 6-cycles of similar adjuvant systemic chemotherapy. Boost irradiation (5.4-9Gy in 3-5 fractions) of metastatic sites was also given to selected patients at the discretion of the treating oncologist. Adjuvant systemic chemotherapy comprised of cisplatin $\left(75 \mathrm{mg} / \mathrm{m}^{2}\right.$ intravenously only on D1 in alternate cycles $2,4,6)$, cyclophosphamide $\left(1000 \mathrm{mg} / \mathrm{m}^{2}\right.$ intravenously on D1 \& D2 in cycles $1,3,5$, and D2 \& D3 in cycles $2,4,6)$ and vincristine $\left(1.5 \mathrm{mg} / \mathrm{m}^{2}\right.$ intravenously on D1 \& D8 in all 6 cycles) administered at 3-weekly intervals. Infants (<3 years) with medulloblastoma were treated with pre-irradiation chemotherapy for 6-12 months or till the age of 3 years (whichever was earlier) followed by risk-stratified CSI plus primary-site boost irradiation as appropriate. After completion of planned treatment, patients were followed-up periodically (3-4 monthly in the first 2-years, 6-monthly until 5-years, and annually thereafter) with annual surveillance MRI scans as per our institutional policy.

\section{Statistical analysis}

The distribution of semantic MRI features amongst the four molecular subgroups was compared using the Pearson chi-square test and Fisher's exact test as appropriate. Relapse (recurrence or progression) was defined as radiographic evidence of new tumor growth or progression of residual tumor on post-treatment follow-up imaging. Relapse-free survival (RFS) was defined as the interval from diagnosis (date of surgery) till documented evidence of clinico-radiological failure (recurrence/progression). Overall survival (OS) was calculated from the date of diagnosis until death from any cause. All time-to event outcomes were analyzed using the product-limit 
method of Kaplan-Meier and compared with the log-rank test for univariate analysis. Semantic imaging features that were deemed statistically significant on univariate testing were entered into a multivariate Cox regression analysis to identify specific imaging features with independent prognostic impact upon survival. Any p-value of $<0.05$ was considered statistically significant. All statistical analysis was done using the Statistical Package for the Social Sciences (SPSS version 21).

\section{Results}

We identified 171 patients of medulloblastoma with pre-operative imaging and known molecular subgroup affiliation at our institute between 2007 and 2018 that comprise the present study cohort. Baseline patient, disease, and treatment-related characteristics across individual molecular subgroups are summarized in Table 1. Briefly, the median age of the study cohort was 9 years with an inter-quartile range (IQR) of 5-16 years with male gender preponderance (3.6:1 ratio). SHH was the most prevalent molecular subgroup $(n=60,35 \%)$ in our study cohort, driven by large number of adult medulloblastomas $(n=40)$, followed by Group $4(n=42,24.5 \%)$, Group $3(n=35,20.5 \%)$, and WNT-pathway $(n=34,20 \%)$ medulloblastoma respectively. The distribution of various semantic MRI features across the four molecular subgroups is described in online supplementary Table S1.

\section{Clinical outcomes}

By the time of this analysis, 55 patients had experienced disease recurrence/progression resulting in a total of 44 deaths, including one death due to treatment-related toxicity (without evidence of disease). The median time to relapse (recurrence/progression) was 19 months (IQR=10-33 months) for the entire study cohort but was significantly different across molecular subgroups. Only one patient with WNT-pathway medulloblastoma had documented recurrence/progression at 9-months from index diagnosis. Time to relapse was significantly longer $(p=0.015)$ in patients with SHH (median 25 months, IQR=12-53 months) and Group 4 (median 23 months, IQR= 16-42 months) medulloblastoma compared to Group 3 tumors (median 10 months, IQR=5-20 months). The pattern of failure was also quite different across molecular subgroups with local tumor-bed recurrence being the predominant mode of first failure in SHH-pathway medulloblastoma, while neuraxial failure with leptomeningeal dissemination being more common in Group 3 and Group 4 tumors (online supplementary Table S2). At a median follow-up of 45 months (IQR=19-65 months), 5-year Kaplan-Meier estimates of RFS and OS with 95\% confidence interval $(\mathrm{Cl})$ were $64 \%(95 \% \mathrm{Cl}: 60-69 \%)$ and 71\% (95\% Cl: 67-75\%) respectively for the entire study cohort (online supplementary file S3). As expected, molecular subgrouping demonstrated prognostic significance for RFS $(p<0.001)$ and OS $(p<0.001)$ on stratified Kaplan-Meier analysis, with WNT-pathway medulloblastoma having the best outcomes while Group 3 medulloblastoma showing the worst survival (online supplementary file S4).

\section{Prognostic impact of MRI features}

Univariate analysis demonstrating prognostic impact of specific semantic imaging features on RFS and OS in all patients across molecular subgroups as well as within induvial subgroups is summarized in Tables 2 and 3 , respectively. Six semantic imaging features significantly impacted upon outcomes - non-central tumor location on vertical axis, absence of brainstem involvement, $\leq 80 \%$ solid tumor area with contrast-uptake, heterogenous pattern of contrast-enhancement, presence of necrosis, and calcification - were all associated with significantly inferior RFS on univariate analysis (online supplementary file S5). Given that only a single WNT patient had experienced relapse, specific imaging features within WNT-subgroup medulloblastoma could not be further correlated with RFS. Within SHH-subgroup medulloblastoma, only non-central tumor location on vertical axis 
predicted significantly inferior RFS. In Group 3 medulloblastoma, five imaging features - non-central tumor location on vertical axis, absence of brainstem involvement, $\leq 80 \%$ solid tumor area with contrast-uptake, presence of necrosis, and calcification - were all associated with significantly worse RFS. Heterogenous pattern of contrast-enhancement was the only factor predicting significantly inferior RFS within Group 4 medulloblastoma. Similarly, six semantic imaging features - non-central tumor location on vertical axis, absence of brainstem involvement, heterogenous pattern of contrast-enhancement, T2-weighted heterogeneity, presence of necrosis, and calcification - were all associated with worse OS on univariate analysis (Figure 1). No deaths were encountered in WNT-subgroup medulloblastoma precluding any correlation of specific imaging features with survival. Within SHH-subgroup medulloblastoma, only non-central tumor location on vertical axis predicted significantly inferior OS. In Group 3 tumors, six semantic imaging features - non-central tumor location on vertical axis, absence of brainstem involvement, $\leq 80 \%$ solid tumor area with contrast-uptake, infiltrative tumor margin, presence of necrosis, and calcification - were all associated with significantly worse OS. Heterogenous pattern of contrast-enhancement and T2-weighted heterogeneity predicted inferior OS within Group 4 medulloblastoma.

Multivariate Cox regression analysis of semantic imaging features for RFS and OS in all patients across all four molecular subgroups is described in Table 4. For the entire study cohort, three imaging features - tumor location on vertical axis $(p=0.029)$, brainstem involvement $(p=0.031)$, and calcification $(p=0.013)$ were associated with independent prognostic significance for RFS. The same three imaging features - tumor location on vertical axis $(p=0.011)$, brainstem involvement $(p=0.022)$, and calcification $(p=0.021)$ were also found to be significant and independent prognostic factors for OS. None of the semantic MRI features were significant within WNT-pathway medulloblastoma and only one imaging feature was significant within SHH-subgroup medulloblastoma on univariate precluding any multivariate analysis, which was limited to Group 3 and Group 4 tumors. For Group 3 tumors, percentage of solid tumor area with contrast-uptake demonstrated independent statistical significance for RFS ( $p=0.009)$ and OS $(p=0.02)$, respectively, while calcification $(p=0.049)$ achieved statistical significance for RFS but not OS. None of the imaging features that correlated significantly with RFS and/or OS in Group 4 medulloblastoma on univariate analysis demonstrated any independent prognostic impact on Cox regression analysis.

\section{Discussion}

Novel biological insights have led to consensus classification of medulloblastoma into four distinct molecular subgroups [6] which are now incorporated in the updated 2016 WHO classification [7] of embryonal CNS tumors. A refined contemporary risk-stratification system including molecular subgrouping [8] assigning medulloblastoma to low-risk (>90\% OS), standard-risk (>75-90\% OS), high-risk (>50-75\% OS), and very high-risk (<50\% OS) categories has largely supplanted the traditional risk-classification system [18] which was based entirely on clinico-radiological features without incorporating histo-molecular information. 'A picture is worth more than a thousand words' - is not just an old adage [19], but a true reflection of the radiomic/radiogenomic revolution in contemporary radiology further fuelled by artificial intelligence approaches.

Molecular subgroups of medulloblastoma have different putative cells of origin $[5,20]$, explaining the varying location of tumor within the posterior fossa. Although we did not find any difference in outcomes based on tumor location on horizontal axis (midline versus lateralized); location of tumor on the vertical axis had a strong prognostic impact on survival. Non-central tumor location (epicentered superiorly or inferiorly) was associated with significantly worse RFS and OS compared to centrally located tumors across molecular subgroups and in

Page $7 / 23$ 
SHH-subgroup and Group 3 medulloblastoma. Another interesting finding from our study was significantly worse outcomes in patients with absence of brainstem involvement compared to tumors with brainstem involvement for the entire cohort and within Group 3 medulloblastoma. The traditional belief that medulloblastoma with brainstem involvement is associated with poorer outcomes (as it precludes gross total resection) was dispelled by a large consortium study [21] which showed that the prognostic benefit of increased extent of resection in medulloblastoma gets largely attenuated after accounting for molecular subgrouping. We also believe that 'cell of origin' drives tumor biology more strongly than technical nuances in neuro-surgery. In adult diffuse gliomas, contrast-enhancement has been an imaging surrogate for histologic grading, with higher grade gliomas showing significantly more enhancement, suggesting blood-brain barrier disruption, increased neovascularization, and transendothelial diffusion [22]. Previous studies have shown differential pattern of contrast-uptake within individual subgroups of medulloblastoma; WNT-pathway tumors are associated with bright homogenous enhancement while Group 4 medulloblastomas have large non-enhancing areas or faint/patchy contrast-uptake $[12,15]$. We found contrast-uptake in $\leq 80 \%$ of tumor volume to be associated with worse outcomes for the entire cohort and within Group 3 medulloblastoma. Patients with heterogeneous pattern of contrast-enhancement demonstrated inferior survival outcomes in the overall cohort and within Group 4 medulloblastoma. We hypothesize that extensive (>80\%) contrast-uptake in medulloblastoma possibly represents favorable biology disease compared to tumors with large non-enhancing tumor areas that may represent degenerative changes and non-proliferative milieu that responds poorly to anti-cancer treatment. Another possible reason for improved survival of patients with bright and uniform contrast-enhancement is the belief that such enhancement is a reflection of blood-brain barrier disruption leading to better permeation of systemic therapy and immunological response compared to tumors with modest/poor contrast-uptake. Contradictory to the findings of our study, Lastowska et al. [23] have shown inferior survival for patients with contrast-uptake in $>75 \%$ of tumor volume in a cohort of non-WNT/non-SHH medulloblastoma. Necrosis has far long been recognized as an adverse prognostic feature in patients with brain tumors and is reported quite frequently in adult diffuse glioma [24]. In the present cohort, radiological evidence of necrosis emerged as a poor prognostic biomarker for the overall cohort as well as within Group 3 tumors. Histo-pathological evidence of tumor necrosis in resected specimens of medulloblastoma has been associated with variable impact on survival. Necrosis on histo-pathology was associated with inferior outcomes in a multi-institutional cohort 79 children with medulloblastoma [25]. On the other hand, presence of necrosis on tumor-tissue blocks did not correlate with outcomes in children with medulloblastoma treated on a large prospective clinical trial [26]. In another study [27] assessing the impact of several histological features on survival in medulloblastoma $(n=556)$, necrosis emerged as a poor prognostic factor; however other histologic features such as calcification and microcysts did not correlate with survival. Intriguingly, we found presence of calcification to be an adverse prognostic factor in medulloblastoma, which traditionally has been recognized as an imaging biomarker of favorable and indolent biology in various brain tumors.

\section{Strengths and limitations:}

Inclusion of 171 patients in our study makes it by far the largest dataset assessing and reporting radiomics/radiogenomics in medulloblastoma. Use of semantic MRI features (visible to the human eye) for correlation with molecular subgrouping and survival makes it easy and simple enough to be adopted in the community without the need for elaborate and sophisticated machine learning algorithms required for radiomicsbased analysis. Semantic imaging features were extracted by consensus in a multi-disciplinary joint clinic improving generalizability and adding robustness. All patients were treated relatively uniformly based on the traditional risk-stratification system and underwent surveillance MRI periodically on follow-up allowing statistical 
correlation of imaging features with outcomes. Despite the aforesaid strengths, several caveats and limitations remain. Our study suffers from all the intrinsic biases and shortcomings of any retrospective analysis. Although our study cohort was large, the number of patients within individual molecular subgroups was relatively small, rendering within subgroup analysis less robust. Imaging features were exclusively derived from T1-weighted (preand post-contrast) and T2-weighted images without taking into account diffusion-weighted imaging, perfusion imaging, and spectroscopy, all of which provide useful and valuable information in medulloblastoma. Finally, median follow-up of our study was relatively short (at less than 4 years); it is well known that late relapses, particularly in adult SHH-subgroup and deaths - including non-cancer mortality [28] can be seen in long-term survivors of medulloblastoma on extended and mature follow-up.

\section{Conclusions}

Imaging features reflect underlying inherent disease biology including the dynamics of complex biological processes rendering them as them useful diagnostic, predictive, and prognostic biomarkers. Distinctive semantic MRI features correlate significantly with survival outcomes in medulloblastoma including within individual molecular subgroups reflecting their prognostic impact that transcends radiogenomic correlation.

\section{Declarations}

Funding: (i) Intramural grant from Tata Memorial Centre, Mumbai; (ii) Indian Council of Medical Research (ICMR), New Delhi; and (iii) Brain Tumor Foundation (BTF) of India, Mumbai. The funding bodies had no influence on the study design, data collection, analysis, interpretation of data, or the manuscript's writing.

\section{Conflict of interest:}

Archya Dasgupta: None

Tejpal Gupta: None

Madan Maitre: None

Babusha Kalra: None

Abhishek Chatterjee: None

Rahul Krishnatry: None

Jayant Sastri Goda: None

Neelam Shirsat: None

Sridhar Epari: None

Ayushi Sahay: None

Amit Janu: None

Sona Pungavkar: None 
Girish Chinnaswamy: None

Vijay Patil: None

Aliasgar Moiyadi: None

Prakash Shetty: None

Rakesh Jalali: None

Availability of data and material: Data will be made available on request to the corresponding author following institutional ethics committee protocols.

Code availability: Not applicable

Author contributions:

Conception and design: TG \& RJ conceptualized the study, and AD wrote the study protocol

Collection and assembly of data: All authors

Data analysis and interpretation: $A D, M M, B K$, and $T G$

Manuscript writing: AD prepared the initial draft, TG edited the final draft manuscript

Final approval of manuscript: All authors

All the authors are in agreement and accountable for all the aspects of the work.

Presentation: Presented in part at the $12^{\text {th }}$ Virtual Annual Conference of Indian Society of Neuro-Oncology (ISNOCON-2021) hosted by Christian Medical College, Vellore, India, in April 2021

Acknowledgments: We acknowledge and thank Nazia Bano, Shraddha Churi, and Farnaz Shaikh for their logistic and secretarial assistance during the conduct of the study. We express our gratitude to all patients and their caregivers who participated in the study.

Conflicts of interest: None of the authors have any conflicts of interest to declare

\section{References}

1. Leece R, Xu J, Ostrom QT et al (2017) Global incidence of malignant brain and other central nervous system tumors by histology, 2003-2007. Neuro-Oncol 19:1553-1564. https://doi.org/10.1093/neuonc/nox091

2. Ostrom QT, Patil N, Cioffi G et al (2020) CBTRUS Statistical Report: Primary Brain and Other Central Nervous System Tumors Diagnosed in the United States in 2013-2017. Neuro-Oncol 22:iv1-iv96. https://doi.org/10.1093/neuonc/noaa200

3. Northcott PA, Korshunov A, Witt H et al (2011) Medulloblastoma Comprises Four Distinct Molecular Variants. J Clin Oncol 29:1408-1414. https://doi.org/10.1200/JC0.2009.27.4324 
4. Gajjar A, Bowers DC, Karajannis MA et al (2015) Pediatric Brain Tumors: Innovative Genomic Information Is Transforming the Diagnostic and Clinical Landscape. J Clin Oncol Off J Am Soc Clin Oncol 33:2986-2998. https://doi.org/10.1200/JC0.2014.59.9217

5. Gupta T, Shirsat N, Jalali R (2015) Molecular Subgrouping of Medulloblastoma: Impact Upon Research and Clinical Practice. Curr Pediatr Rev 11:106-119. https://doi.org/10.2174/1573396311666150702104030

6. Taylor MD, Northcott PA, Korshunov A et al (2012) Molecular subgroups of medulloblastoma: the current consensus. Acta Neuropathol (Berl) 123:465-472. https://doi.org/10.1007/s00401-011-0922-z

7. Louis DN, Perry A, Reifenberger G et al (2016) The 2016 World Health Organization Classification of Tumors of the Central Nervous System: a summary. Acta Neuropathol (Berl) 131:803-820.

https://doi.org/10.1007/s00401-016-1545-1

8. Ramaswamy V, Remke M, Bouffet E et al (2016) Risk stratification of childhood medulloblastoma in the molecular era: the current consensus. Acta Neuropathol (Berl) 131:821-831.

https://doi.org/10.1007/s00401-016-1569-6

9. Kuo MD, Jamshidi N (2014) Behind the numbers: Decoding molecular phenotypes with radiogenomicsguiding principles and technical considerations. Radiology 270:320-325.

https://doi.org/10.1148/radiol.13132195

10. Gillies RJ, Kinahan PE, Hricak H (2015) Radiomics: Images Are More than Pictures, They Are Data. Radiology 278:563-577. https://doi.org/10.1148/radiol.2015151169

11. Kickingereder P, Andronesi OC (2018) Radiomics, Metabolic, and Molecular MRI for Brain Tumors. Semin Neurol 38:32-40. https://doi.org/10.1055/s-0037-1618600

12. Dasgupta A, Gupta T, Pungavkar S et al (2019) Nomograms based on preoperative multiparametric magnetic resonance imaging for prediction of molecular subgrouping in medulloblastoma: results from a radiogenomics study of 111 patients. Neuro-Oncol 21:115-124. https://doi.org/10.1093/neuonc/noy093

13. Iv M, Zhou M, Shpanskaya K et al (2019) MR Imaging-Based Radiomic Signatures of Distinct Molecular Subgroups of Medulloblastoma. AJNR Am J Neuroradiol 40:154-161. https://doi.org/10.3174/ajnr.A5899

14. Yan J, Liu L, Wang W et al (2020) Radiomic Features From Multi-Parameter MRI Combined With Clinical Parameters Predict Molecular Subgroups in Patients With Medulloblastoma. Front Oncol 10:558162. https://doi.org/10.3389/fonc.2020.558162

15. Dasgupta A, Gupta T (2018) Radiogenomics of medulloblastoma: imaging surrogates of molecular biology. J Transl Genet Genomics 2:. https://doi.org/10.20517/jtgg.2018.21

16. Colafati GS, Voicu IP, Carducci C et al (2018) MRI features as a helpful tool to predict the molecular subgroups of medulloblastoma: state of the art. Ther Adv Neurol Disord 11:. https://doi.org/10.1177/1756286418775375

17. Kunder R, Jalali R, Sridhar E et al (2013) Real-time PCR assay based on the differential expression of microRNAs and protein-coding genes for molecular classification of formalin-fixed paraffin embedded medulloblastomas. Neuro-Oncol 15:1644-1651. https://doi.org/10.1093/neuonc/not123

18. Zeltzer PM, Boyett JM, Finlay JL et al (1999) Metastasis stage, adjuvant treatment, and residual tumor are prognostic factors for medulloblastoma in children: conclusions from the Children's Cancer Group 921 randomized phase III study. J Clin Oncol Off J Am Soc Clin Oncol 17:832-845.

https://doi.org/10.1200/JC0.1999.17.3.832

Page $11 / 23$ 
19. Rabasco Meneghetti A, Zwanenburg A, Löck S (2020) Pictures worth more than a thousand words: Prediction of survival in medulloblastoma patients. EBioMedicine 62:103136.

https://doi.org/10.1016/j.ebiom.2020.103136

20. Gibson P, Tong Y, Robinson G et al (2010) Subtypes of medulloblastoma have distinct developmental origins. Nature 468:1095-1099. https://doi.org/10.1038/nature09587

21. Thompson EM, Hielscher T, Bouffet E et al (2016) Prognostic value of medulloblastoma extent of resection after accounting for molecular subgroup: a retrospective integrated clinical and molecular analysis. Lancet Oncol 17:484-495. https://doi.org/10.1016/S1470-2045(15)00581-1

22. Law M, Yang S, Babb JS et al (2004) Comparison of cerebral blood volume and vascular permeability from dynamic susceptibility contrast-enhanced perfusion MR imaging with glioma grade. AJNR Am J Neuroradiol $25: 746-755$

23. Łastowska M, Jurkiewicz E, Trubicka J et al (2015) Contrast enhancement pattern predicts poor survival for patients with non-WNT/SHH medulloblastoma tumours. J Neurooncol 123:65-73.

https://doi.org/10.1007/s11060-015-1779-0

24. Liu S, Wang Y, Xu K et al (2017) Relationship between necrotic patterns in glioblastoma and patient survival: fractal dimension and lacunarity analyses using magnetic resonance imaging. Sci Rep 7: https://doi.org/10.1038/s41598-017-08862-6

25. Urberuaga A, Navajas A, Burgos J, Pijoán Jl (2006) A review of clinical and histological features of Spanish paediatric medulloblastomas during the last 21 years. Childs Nerv Syst ChNS Off J Int Soc Pediatr Neurosurg 22:466-474. https://doi.org/10.1007/s00381-005-0004-4

26. Giangaspero F, Wellek S, Masuoka J et al (2006) Stratification of medulloblastoma on the basis of histopathological grading. Acta Neuropathol (Berl) 112:5-12. https://doi.org/10.1007/s00401-006-0064-x

27. Verma S, Tavaré CJ, Gilles FH (2008) Histologic features and prognosis in pediatric medulloblastoma. Pediatr Dev Pathol Off J Soc Pediatr Pathol Paediatr Pathol Soc 11:337-343. https://doi.org/10.2350/07-090353.1

28. Salloum R, Chen Y, Yasui Y et al (2019) Late Morbidity and Mortality Among Medulloblastoma Survivors Diagnosed Across Three Decades: A Report From the Childhood Cancer Survivor Study. J Clin Oncol Off J Am Soc Clin Oncol 37:731-740. https://doi.org/10.1200/JC0.18.00969

\section{Tables}


Table 1

Baseline patient, disease, and treatment-related characteristics of the study cohort $(\mathrm{N}=171)$

\begin{tabular}{|c|c|c|c|c|c|}
\hline Features & WNT $(n=34)$ & $\mathrm{SHH}(n=60)$ & Group 3 (n = 35) & Group $4(n=42)$ & $\mathrm{p}$ value \\
\hline \multicolumn{6}{|l|}{ Age } \\
\hline$<3$ years & $0(0 \%)$ & $8(13 \%)$ & $12(34 \%)$ & $1(2 \%)$ & \\
\hline $3-18$ years & 27 (79\%) & $23(38 \%)$ & 20 (57\%) & 40 (96\%) & $<0.001$ \\
\hline$>18$ years & $7(21 \%)$ & 29 (49\%) & $3(9 \%)$ & $1(2 \%)$ & \\
\hline \multicolumn{6}{|l|}{ Gender } \\
\hline Male & $24(71 \%)$ & $43(72 \%)$ & $28(80 \%)$ & 39 (93\%) & 0.045 \\
\hline Female & $10(29 \%)$ & $17(28 \%)$ & $7(20 \%)$ & $3(7 \%)$ & \\
\hline \multicolumn{6}{|l|}{ Histology } \\
\hline Classic & $26(77 \%)$ & 19 (32\%) & $18(51 \%)$ & $22(52 \%)$ & \\
\hline Desmoplastic & $0(0 \%)$ & 27 (45\%) & $3(9 \%)$ & $10(24 \%)$ & \\
\hline Large cell/ anaplastic & $0(0 \%)$ & $8(13 \%)$ & $11(31 \%)$ & $2(5 \%)$ & $<0.001$ \\
\hline MBEN & $0(0 \%)$ & $3(5 \%)$ & $0(0 \%)$ & $(0 \%)$ & \\
\hline Others/ unclassified & $8(23 \%)$ & $3(5 \%)$ & $3(9 \%)$ & $8(19 \%)$ & \\
\hline \multicolumn{6}{|l|}{ Metastatic disease ${ }^{a}$} \\
\hline Present & $6(18 \%)$ & $14(23 \%)$ & $20(57 \%)$ & $15(36 \%)$ & $<0.001$ \\
\hline Absent & $28(82 \%$ & $46(77 \%)$ & $15(43 \%)$ & 27 (64\%) & \\
\hline \multicolumn{6}{|l|}{ Residual disease } \\
\hline$<1.5 \mathrm{~cm}^{2}$ & $26(77 \%)$ & $39(65 \%)$ & $25(71 \%)$ & $31(74 \%)$ & \\
\hline $1.5 \mathrm{~cm}^{2}$ or more & $8(24 \%)$ & $19(32 \%)$ & $10(29 \%)$ & $8(19 \%)$ & 0.343 \\
\hline Unknown & $0(0 \%)$ & $2(3 \%)$ & $0(0 \%)$ & $3(7 \%)$ & \\
\hline \multicolumn{6}{|l|}{ Risk-stratification } \\
\hline Standard risk & $23(68 \%)$ & $25(42 \%)$ & $9(26 \%)$ & $18(43 \%)$ & \\
\hline High risk & $11(32 \%)$ & $35(58 \%)$ & $26(74 \%)$ & $22(52 \%)$ & 0.004 \\
\hline Unknown & $0(0 \%)$ & $0(0 \%)$ & $0(0 \%)$ & $2(5 \%)$ & \\
\hline \multicolumn{6}{|l|}{ Dose of CSI } \\
\hline$\geq 3000$ cGy & $23(68 \%)$ & 47 (79\%) & $21(60 \%)$ & 27 (64\%) & \\
\hline 2340 cGy & $11(32 \%)$ & $12(20 \%)$ & $7(20 \%)$ & $13(31 \%)$ & 0.003 \\
\hline Unknown & $0(0 \%)$ & $0(0 \%)^{b}$ & $7(20 \%)$ & $2(5 \%)$ & \\
\hline
\end{tabular}




\begin{tabular}{|llllll|}
\hline Features & WNT $(\mathbf{n = 3 4 )}$ & SHH $(\mathbf{n = 6 0 )}$ & Group 3 $(\mathbf{n = 3 5 )}$ & Group 4 (n= 42) & p value \\
\hline Chemotherapyc $^{c}$ & & & & \\
\hline Yes & $29(85 \%)$ & $53(88 \%)$ & $23(66 \%)$ & $39(93 \%)$ & 0.007 \\
\hline No & $5(15 \%)$ & $7(12 \%)$ & $12(34 \%)$ & $3(7 \%)$ & \\
\hline
\end{tabular}

MBEN=Medulloblastoma with extensive nodularity; CSI=Cranio-spinal irradiation

a metastatic disease at presentation

${ }^{b}$ one patient with $\mathrm{SHH}$ had received focal radiation to the posterior fossa without CSI

${ }^{c}$ Upfront chemotherapy at initial diagnosis (concurrent/ adjuvant)

All statistically significant $\mathrm{p}$-values $(<0.05)$ are highlighted in bold 
Table 2

Prognostic impact of semantic imaging features on relapse-free survival

\begin{tabular}{|c|c|c|c|c|c|c|c|c|c|c|}
\hline \multirow[t]{2}{*}{ Features } & \multicolumn{2}{|c|}{ All patients } & \multicolumn{2}{|l|}{ WNT } & \multicolumn{2}{|l|}{ SHH } & \multicolumn{2}{|c|}{ Group 3} & \multicolumn{2}{|c|}{ Group 4} \\
\hline & $\begin{array}{l}5-\mathrm{yr} \\
\text { RFS }\end{array}$ & $\begin{array}{l}\mathrm{p}- \\
\text { value }\end{array}$ & $\begin{array}{l}5-y r \\
\text { RFS }\end{array}$ & $\begin{array}{l}p- \\
\text { value }\end{array}$ & $\begin{array}{l}5-\mathrm{yr} \\
\text { RFS }\end{array}$ & $\begin{array}{l}\mathrm{p}- \\
\text { value }\end{array}$ & $\begin{array}{l}5-y r \\
\text { RFS }\end{array}$ & $\begin{array}{l}p- \\
\text { value }\end{array}$ & $\begin{array}{l}5-y r \\
\text { RFS }\end{array}$ & $\begin{array}{l}p- \\
\text { value }\end{array}$ \\
\hline \multicolumn{11}{|l|}{ Location-horizontal } \\
\hline Midline & $63 \%$ & 0.257 & $100 \%$ & 0.643 & $74 \%$ & 0.693 & $43 \%$ & NA & $50 \%$ & NA \\
\hline Lateral & $70 \%$ & & $96 \%$ & & $66 \%$ & & NA & & NA & \\
\hline \multicolumn{11}{|l|}{ Tumor-vertical } \\
\hline Superior & $63 \%$ & & $100 \%$ & & $63 \%$ & & $0 \%$ & & NA & \\
\hline Central & $74 \%$ & 0.002 & $97 \%$ & 0.917 & $87 \%$ & 0.014 & $53 \%$ & 0.011 & $48 \%$ & 0.992 \\
\hline Inferior & $47 \%$ & & $100 \%$ & & $25 \%$ & & $37 \%$ & & $48 \%$ & \\
\hline \multicolumn{11}{|l|}{$\begin{array}{l}\text { Brainstem } \\
\text { involvement }\end{array}$} \\
\hline Involved & $71 \%$ & 0.009 & $97 \%$ & 0.715 & $75 \%$ & 0.332 & $60 \%$ & 0.006 & $51 \%$ & 0.774 \\
\hline Uninvolved & $53 \%$ & & $100 \%$ & & $64 \%$ & & $20 \%$ & & $0 \%$ & \\
\hline \multicolumn{11}{|l|}{$\begin{array}{l}\text { Contrast uptake } \\
\text { (area) }\end{array}$} \\
\hline$\leq 80 \%$ & $55 \%$ & $0 . .009$ & $94 \%$ & 0.317 & $65 \%$ & 0.816 & $18 \%$ & $<.001$ & $51 \%$ & 0.448 \\
\hline$>80 \%$ & $81 \%$ & & $100 \%$ & & $73 \%$ & & $85 \%$ & & $33 \%$ & \\
\hline \multicolumn{11}{|l|}{$\begin{array}{l}\text { Contrast } \\
\text { heterogeneity }\end{array}$} \\
\hline Homogeneous & $76 \%$ & 0.026 & $95 \%$ & 0.403 & $69 \%$ & 0.478 & $67 \%$ & 0.222 & $0 \%$ & $\stackrel{<}{0.001}$ \\
\hline Heterogeneous & $56 \%$ & & $100 \%$ & & $67 \%$ & & $36 \%$ & & $45 \%$ & \\
\hline \multicolumn{11}{|l|}{ T2W intensity } \\
\hline Isointense & $71 \%$ & 0.066 & $96 \%$ & 0.489 & $78 \%$ & 0.066 & $50 \%$ & 0.203 & $53 \%$ & 0.577 \\
\hline $\begin{array}{l}\text { Others } \\
\text { (Hypo/hyperintense) }\end{array}$ & $54 \%$ & & $100 \%$ & & $52 \%$ & & $27 \%$ & & $49 \%$ & \\
\hline \multicolumn{11}{|l|}{ T2W homogeneity } \\
\hline Homogeneous & $70 \%$ & 0.173 & $96 \%$ & 0.549 & $70 \%$ & 0.573 & $50 \%$ & 0.519 & $61 \%$ & 0.193 \\
\hline Heterogeneous & $57 \%$ & & $100 \%$ & & $68 \%$ & & $31 \%$ & & $31 \%$ & \\
\hline \multicolumn{11}{|l|}{ Tumor margin } \\
\hline Smooth & $62 \%$ & & $100 \%$ & & $70 \%$ & & $42 \%$ & & $54 \%$ & \\
\hline
\end{tabular}




\begin{tabular}{|c|c|c|c|c|c|c|c|c|c|c|}
\hline \multirow{2}{*}{$\begin{array}{l}\text { Features } \\
\text { Infiltrative }\end{array}$} & \multicolumn{2}{|c|}{ All patients } & \multicolumn{2}{|l|}{ WNT } & \multicolumn{2}{|l|}{$\mathrm{SHH}$} & \multicolumn{2}{|c|}{ Group 3} & \multicolumn{2}{|c|}{ Group 4} \\
\hline & $61 \%$ & 0.562 & $91 \%$ & 0.352 & $60 \%$ & 0.691 & $0 \%$ & 0.120 & $100 \%$ & 0.397 \\
\hline Lobulated & $67 \%$ & & $100 \%$ & & $70 \%$ & & $59 \%$ & & $38 \%$ & \\
\hline \multicolumn{11}{|c|}{ Peritumoral edema } \\
\hline Present & $69 \%$ & 0.296 & $100 \%$ & 0.611 & $69 \%$ & 0.956 & $50 \%$ & 0.793 & $57 \%$ & 0.909 \\
\hline Absent & $61 \%$ & & $96 \%$ & & $80 \%$ & & $41 \%$ & & $49 \%$ & \\
\hline \multicolumn{11}{|c|}{ Hemorrhage } \\
\hline Present & $79 \%$ & 0.246 & $100 \%$ & 0.607 & $100 \%$ & 0.438 & $0 \%$ & 0.227 & $83 \%$ & 0.933 \\
\hline Absent & $62 \%$ & & $94 \%$ & & $68 \%$ & & $47 \%$ & & $49 \%$ & \\
\hline \multicolumn{11}{|l|}{ Necrosis } \\
\hline Present & $28 \%$ & $\begin{array}{l}< \\
0.001\end{array}$ & $100 \%$ & 0.862 & $36 \%$ & 0.222 & $0 \%$ & $\begin{array}{l}< \\
0.001\end{array}$ & $57 \%$ & 0.916 \\
\hline Absent & $69 \%$ & & $97 \%$ & & $72 \%$ & & $56 \%$ & & $49 \%$ & \\
\hline \multicolumn{11}{|c|}{ Calcification } \\
\hline Present & $55 \%$ & 0.018 & $100 \%$ & 0.593 & $71 \%$ & 0.988 & $27 \%$ & 0.014 & $58 \%$ & 0.826 \\
\hline Absent & $67 \%$ & & $95 \%$ & & $66 \%$ & & $67 \%$ & & $44 \%$ & \\
\hline \multicolumn{11}{|c|}{ Cyst number } \\
\hline Absent & $70 \%$ & & $100 \%$ & & $71 \%$ & & $56 \%$ & & $86 \%$ & \\
\hline $1-3$ & $75 \%$ & 0.949 & $100 \%$ & 0.835 & $100 \%$ & 0.333 & $42 \%$ & 0.437 & $75 \%$ & 0.347 \\
\hline$>3$ & $62 \%$ & & $96 \%$ & & $67 \%$ & & $36 \%$ & & $38 \%$ & \\
\hline \multicolumn{11}{|l|}{ Cyst size } \\
\hline Microcyst & $72 \%$ & & $100 \%$ & & $74 \%$ & & $50 \%$ & & $53 \%$ & \\
\hline Macrocyst & $75 \%$ & 0.332 & $100 \%$ & 0.355 & $100 \%$ & 0.728 & $75 \%$ & 0.264 & $0 \%$ & 0.580 \\
\hline Both & $43 \%$ & & $88 \%$ & & $55 \%$ & & $13 \%$ & & $0 \%$ & \\
\hline \multicolumn{11}{|c|}{ Location of cyst } \\
\hline Peripheral & $88 \%$ & & $100 \%$ & & $100 \%$ & & $67 \%$ & & NA & \\
\hline Central & $67 \%$ & 0.343 & $100 \%$ & 0.355 & $76 \%$ & 0.438 & $47 \%$ & 0.407 & $44 \%$ & 0.485 \\
\hline Both & $48 \%$ & & $88 \%$ & & $54 \%$ & & $17 \%$ & & $0 \%$ & \\
\hline \multicolumn{11}{|c|}{ Hydrocephalus } \\
\hline Present & $62 \%$ & 0.688 & $96 \%$ & 0.611 & $66 \%$ & 0.718 & $45 \%$ & 0.490 & $50 \%$ & NA \\
\hline Absent & $82 \%$ & & $100 \%$ & & $100 \%$ & & $25 \%$ & & NA & \\
\hline
\end{tabular}

RFS: Relapse-free survival; NA: Not applicable (no patients in the group/ no values for comparison) 
All statistically significant p-values $(<0.05)$ are highlighted in bold 
Table 3

Influence of semantic imaging features on overall survival

\begin{tabular}{|c|c|c|c|c|c|c|c|c|c|c|}
\hline \multirow[t]{2}{*}{ Features } & \multicolumn{2}{|c|}{ All patients } & \multicolumn{2}{|l|}{ WNT } & \multicolumn{2}{|l|}{ SHH } & \multicolumn{2}{|c|}{ Group 3} & \multicolumn{2}{|c|}{ Group 4} \\
\hline & $\begin{array}{l}5-y r \\
\text { OS }\end{array}$ & $\begin{array}{l}\mathrm{p}- \\
\text { value }\end{array}$ & $\begin{array}{l}5-y r \\
\text { OS }\end{array}$ & $\begin{array}{l}\mathrm{p}- \\
\text { value }\end{array}$ & $\begin{array}{l}5-y r \\
\text { OS }\end{array}$ & $\begin{array}{l}\mathrm{p}- \\
\text { value }\end{array}$ & $\begin{array}{l}5-y r \\
\text { OS }\end{array}$ & $\begin{array}{l}\mathrm{p}- \\
\text { value }\end{array}$ & $\begin{array}{l}5-y r \\
\text { OS }\end{array}$ & $\begin{array}{l}\mathrm{p}- \\
\text { value }\end{array}$ \\
\hline \multicolumn{11}{|l|}{ Location-horizontal } \\
\hline Midline & $71 \%$ & 0.370 & $100 \%$ & NA & $83 \%$ & 0.933 & $46 \%$ & NA & $67 \%$ & NA \\
\hline Lateral & $75 \%$ & & $100 \%$ & & $73 \%$ & & NA & & NA & \\
\hline \multicolumn{11}{|l|}{ Tumor-vertical } \\
\hline Superior & $65 \%$ & & $100 \%$ & & $65 \%$ & & $0 \%$ & & NA & \\
\hline Central & $84 \%$ & 0.003 & $100 \%$ & NA & $95 \%$ & 0.045 & $60 \%$ & $<0.001$ & $70 \%$ & 0.734 \\
\hline Inferior & $55 \%$ & & $100 \%$ & & $50 \%$ & & $37 \%$ & & $62 \%$ & \\
\hline \multicolumn{11}{|l|}{$\begin{array}{l}\text { Brainstem } \\
\text { involvement }\end{array}$} \\
\hline Involved & $77 \%$ & 0.018 & $100 \%$ & NA & $81 \%$ & 0.462 & $65 \%$ & 0.003 & $63 \%$ & 0.226 \\
\hline Uninvolved & $62 \%$ & & $100 \%$ & & $72 \%$ & & $20 \%$ & & $100 \%$ & \\
\hline \multicolumn{11}{|l|}{$\begin{array}{l}\text { Contrast uptake } \\
\text { (area) }\end{array}$} \\
\hline$\leq 80 \%$ & $68 \%$ & $0 . .061$ & $100 \%$ & NA & $78 \%$ & 0.983 & $23 \%$ & $<0.001$ & $73 \%$ & 0.198 \\
\hline$>80 \%$ & $79 \%$ & & $100 \%$ & & $73 \%$ & & $85 \%$ & & $33 \%$ & \\
\hline \multicolumn{11}{|l|}{$\begin{array}{l}\text { Contrast } \\
\text { heterogeneity }\end{array}$} \\
\hline Homogeneous & $83 \%$ & 0.042 & $100 \%$ & NA & $80 \%$ & 0.311 & $67 \%$ & 0.210 & $0 \%$ & 0.002 \\
\hline Heterogeneous & $65 \%$ & & $100 \%$ & & $67 \%$ & & $39 \%$ & & $69 \%$ & \\
\hline \multicolumn{11}{|l|}{ T2W intensity } \\
\hline Isointense & $75 \%$ & 0.292 & $100 \%$ & NA & $80 \%$ & 0.433 & $50 \%$ & 0.269 & $73 \%$ & 0.525 \\
\hline $\begin{array}{l}\text { Others } \\
\text { (Hypo/hyperintense) }\end{array}$ & $66 \%$ & & $100 \%$ & & $68 \%$ & & $37 \%$ & & $62 \%$ & \\
\hline \multicolumn{11}{|l|}{ T2W homogeneity } \\
\hline Homogeneous & $80 \%$ & 0.030 & $100 \%$ & NA & $85 \%$ & 0.133 & $50 \%$ & 0.606 & $83 \%$ & 0.042 \\
\hline Heterogeneous & $61 \%$ & & $100 \%$ & & $71 \%$ & & $39 \%$ & & $44 \%$ & \\
\hline \multicolumn{11}{|l|}{ Tumor margin } \\
\hline Smooth & $74 \%$ & & $100 \%$ & & $84 \%$ & & $50 \%$ & & $75 \%$ & \\
\hline
\end{tabular}




\begin{tabular}{|c|c|c|c|c|c|c|c|c|c|c|}
\hline \multirow{2}{*}{$\begin{array}{l}\text { Features } \\
\text { Infiltrative }\end{array}$} & \multicolumn{2}{|c|}{ All patients } & \multicolumn{2}{|l|}{ WNT } & \multicolumn{2}{|l|}{$\mathrm{SHH}$} & \multicolumn{2}{|c|}{ Group 3} & \multicolumn{2}{|c|}{ Group 4} \\
\hline & $62 \%$ & 0.328 & $100 \%$ & NA & $60 \%$ & 0.540 & $0 \%$ & 0.016 & $100 \%$ & 0.605 \\
\hline Lobulated & $73 \%$ & & $100 \%$ & & $77 \%$ & & $59 \%$ & & $59 \%$ & \\
\hline \multicolumn{11}{|c|}{ Peritumoral edema } \\
\hline Present & $73 \%$ & 0.643 & $100 \%$ & NA & $76 \%$ & 0.874 & $50 \%$ & 0.736 & $63 \%$ & 0.294 \\
\hline Absent & $71 \%$ & & $100 \%$ & & $80 \%$ & & $45 \%$ & & $72 \%$ & \\
\hline \multicolumn{11}{|c|}{ Hemorrhage } \\
\hline Present & $67 \%$ & 0.693 & $100 \%$ & NA & $100 \%$ & 0.486 & $0 \%$ & 0.167 & $0 \%$ & 0.665 \\
\hline Absent & $71 \%$ & & $100 \%$ & & $75 \%$ & & $50 \%$ & & $71 \%$ & \\
\hline \multicolumn{11}{|l|}{ Necrosis } \\
\hline Present & $50 \%$ & $\begin{array}{l}< \\
0.001\end{array}$ & $100 \%$ & NA & $69 \%$ & 0.178 & $0 \%$ & $\begin{array}{l}< \\
0.001\end{array}$ & $86 \%$ & 0.694 \\
\hline Absent & $75 \%$ & & $100 \%$ & & $77 \%$ & & $59 \%$ & & $65 \%$ & \\
\hline \multicolumn{11}{|c|}{ Calcification } \\
\hline Present & $55 \%$ & 0.006 & $100 \%$ & NA & $69 \%$ & 0.791 & $33 \%$ & 0.044 & $55 \%$ & 0.205 \\
\hline Absent & $77 \%$ & & $100 \%$ & & $75 \%$ & & $67 \%$ & & $69 \%$ & \\
\hline \multicolumn{11}{|c|}{ Cyst number } \\
\hline Absent & $69 \%$ & & $100 \%$ & & $71 \%$ & & $56 \%$ & & $80 \%$ & \\
\hline $1-3$ & $77 \%$ & 0.799 & $100 \%$ & NA & $100 \%$ & 0.300 & $42 \%$ & 0.522 & $100 \%$ & 0.426 \\
\hline$>3$ & $72 \%$ & & $100 \%$ & & $75 \%$ & & $43 \%$ & & $60 \%$ & \\
\hline \multicolumn{11}{|l|}{ Cyst size } \\
\hline Microcyst & $79 \%$ & & $100 \%$ & & $75 \%$ & & $76 \%$ & & $76 \%$ & \\
\hline Macrocyst & $88 \%$ & 0.307 & $100 \%$ & NA & $100 \%$ & 0.512 & $100 \%$ & 0.594 & $100 \%$ & 0.594 \\
\hline Both & $58 \%$ & & $100 \%$ & & $65 \%$ & & $27 \%$ & & $27 \%$ & \\
\hline \multicolumn{11}{|c|}{ Location of cyst } \\
\hline Peripheral & $88 \%$ & & $100 \%$ & & $100 \%$ & & $67 \%$ & & NA & \\
\hline Central & $75 \%$ & 0.590 & $100 \%$ & NA & $79 \%$ & 0.804 & $47 \%$ & 0.741 & $70 \%$ & 0.891 \\
\hline Both & $64 \%$ & & $100 \%$ & & $73 \%$ & & $33 \%$ & & $37 \%$ & \\
\hline \multicolumn{11}{|c|}{ Hydrocephalus } \\
\hline Present & $70 \%$ & 0.797 & $100 \%$ & NA & $74 \%$ & 0.423 & $48 \%$ & 0.365 & $67 \%$ & NA \\
\hline Absent & $82 \%$ & & $100 \%$ & & $100 \%$ & & $25 \%$ & & NA & \\
\hline
\end{tabular}

OS: Overall survival; NA: Not applicable (no patients in the group/ no values for comparison/ no events) 
All statistically significant p-values $(<0.05)$ are highlighted in bold 
Table 4

Multivariate Cox regression analysis of imaging features deemed significant $(p-v a l u e<0.05)$ for relapse-free survival and overall survival on univariate analysis

\begin{tabular}{|c|c|c|c|c|c|c|}
\hline \multirow[t]{2}{*}{ Features } & \multicolumn{3}{|c|}{ Relapse-free survival } & \multicolumn{3}{|c|}{ Overall survival } \\
\hline & $\mathrm{HR}$ & $95 \% \mathrm{Cl}$ & p-value & $\mathrm{HR}$ & $95 \% \mathrm{Cl}$ & p-value \\
\hline \multicolumn{7}{|l|}{ All patients } \\
\hline \multicolumn{7}{|l|}{ Location vertical } \\
\hline Central & 0.45 & $0.22-0.92$ & 0.029 & 0.32 & $0.13-0.77$ & 0.011 \\
\hline Superior & 1.06 & $0.44-2.59$ & 0.889 & 0.88 & $0.31-2.51$ & 0.817 \\
\hline Inferior & Reference & & & Reference & & \\
\hline \multicolumn{7}{|l|}{ Brainstem } \\
\hline Involved & 0.48 & $0.25-0.94$ & 0.031 & 0.41 & $0.19-0.88$ & 0.022 \\
\hline Uninvolved & Reference & & & Reference & & \\
\hline \multicolumn{7}{|l|}{ Contrast-area } \\
\hline$\leq 80 \%$ & 2.06 & $0.93-4.60$ & 0.076 & & NA & \\
\hline$>80 \%$ & Reference & & & & & \\
\hline \multicolumn{7}{|c|}{ Contrast-heterogeneity } \\
\hline Homogeneous & 0.71 & $0.30-1.67$ & 0.425 & 0.48 & $0.19-1.17$ & 0.106 \\
\hline Heterogeneous & Reference & & & Reference & & \\
\hline \multicolumn{7}{|c|}{ T2W homogeneity } \\
\hline Homogeneous & NA & & & 0.61 & $0.29-1.31$ & 0.205 \\
\hline Heterogeneous & & & & Reference & & \\
\hline \multicolumn{7}{|l|}{ Necrosis } \\
\hline Present & 1.48 & $0.70-31.6$ & 0.305 & 1.28 & $0.57-2.88$ & 0.554 \\
\hline Absent & & & & Reference & & \\
\hline \multicolumn{7}{|l|}{ Calcification } \\
\hline Present & 2.41 & $1.21-4.83$ & 0.013 & 2.44 & $1.15-5.20$ & 0.021 \\
\hline Absent & Reference & & & Reference & & \\
\hline \multicolumn{7}{|l|}{ Group 3} \\
\hline \multicolumn{7}{|c|}{ Location vertical } \\
\hline Central & 0.86 & $0.22-3.36$ & 0.823 & 0.99 & $0.26-4.37$ & 0.991 \\
\hline Superior & 12.91 & $0.89-187.10$ & 0.061 & 13.2 & $0.01-245.12$ & 0.924 \\
\hline
\end{tabular}




\begin{tabular}{|c|c|c|c|c|c|c|}
\hline Features & Relapse-free & survival & & Overall surv & ival & \\
\hline Inferior & Reference & & & Reference & & \\
\hline \multicolumn{7}{|l|}{ Brainstem } \\
\hline Involved & 0.44 & $0.10-1.86$ & 0.262 & 0.49 & $0.10-2.44$ & 0.385 \\
\hline Uninvolved & Reference & & & Reference & & \\
\hline \multicolumn{7}{|l|}{ Contrast-area } \\
\hline$\leq 80 \%$ & 17.67 & $2.06-151.81$ & 0.009 & 12.23 & $1.49-100.51$ & 0.020 \\
\hline$>80 \%$ & Reference & & & Reference & & \\
\hline \multicolumn{7}{|l|}{ Tumor margin } \\
\hline Smooth & NA & & & 1.02 & $0.28-3.66$ & 0.977 \\
\hline Infiltrative & & & & 0.01 & $0.01-113.32$ & 0.933 \\
\hline Lobulated & & & & Reference & & \\
\hline \multicolumn{7}{|l|}{ Necrosis } \\
\hline Present & 1.15 & $0.24-5.42$ & 0.860 & Reference & & \\
\hline Absent & Reference & & & 0.01 & $<0.01-115.91$ & 0.925 \\
\hline \multicolumn{7}{|l|}{ Calcification } \\
\hline Present & 4.05 & $1.01-16.34$ & 0.049 & 2.30 & $0.51-10.34$ & 0.279 \\
\hline Absent & Reference & & & Reference & & \\
\hline \multicolumn{7}{|l|}{ Group 4} \\
\hline \multicolumn{7}{|c|}{ Contrast-heterogeneity } \\
\hline Homogeneous & & NA & & 7.08 & $0.60-83.92$ & 0.121 \\
\hline Heterogeneous & & & & Reference & & \\
\hline \multicolumn{7}{|c|}{ T2W Homogeneity } \\
\hline Homogeneous & & NA & & 0.25 & $0.06-1.11$ & 0.069 \\
\hline Heterogeneous & & & & Reference & & \\
\hline
\end{tabular}

MVA: Multivariate analysis; HR: Hazard ratio; Cl: Confidence Interval; NA: Not applicable

Multivariate Cox regression analysis was not performed due to absence of (within WNT subgroup) or one (within SHH subgroup) significant feature on univariate analysis.

All statistically significant $\mathrm{p}$-values $(<0.05)$ are highlighted in bold.

\section{Figures}



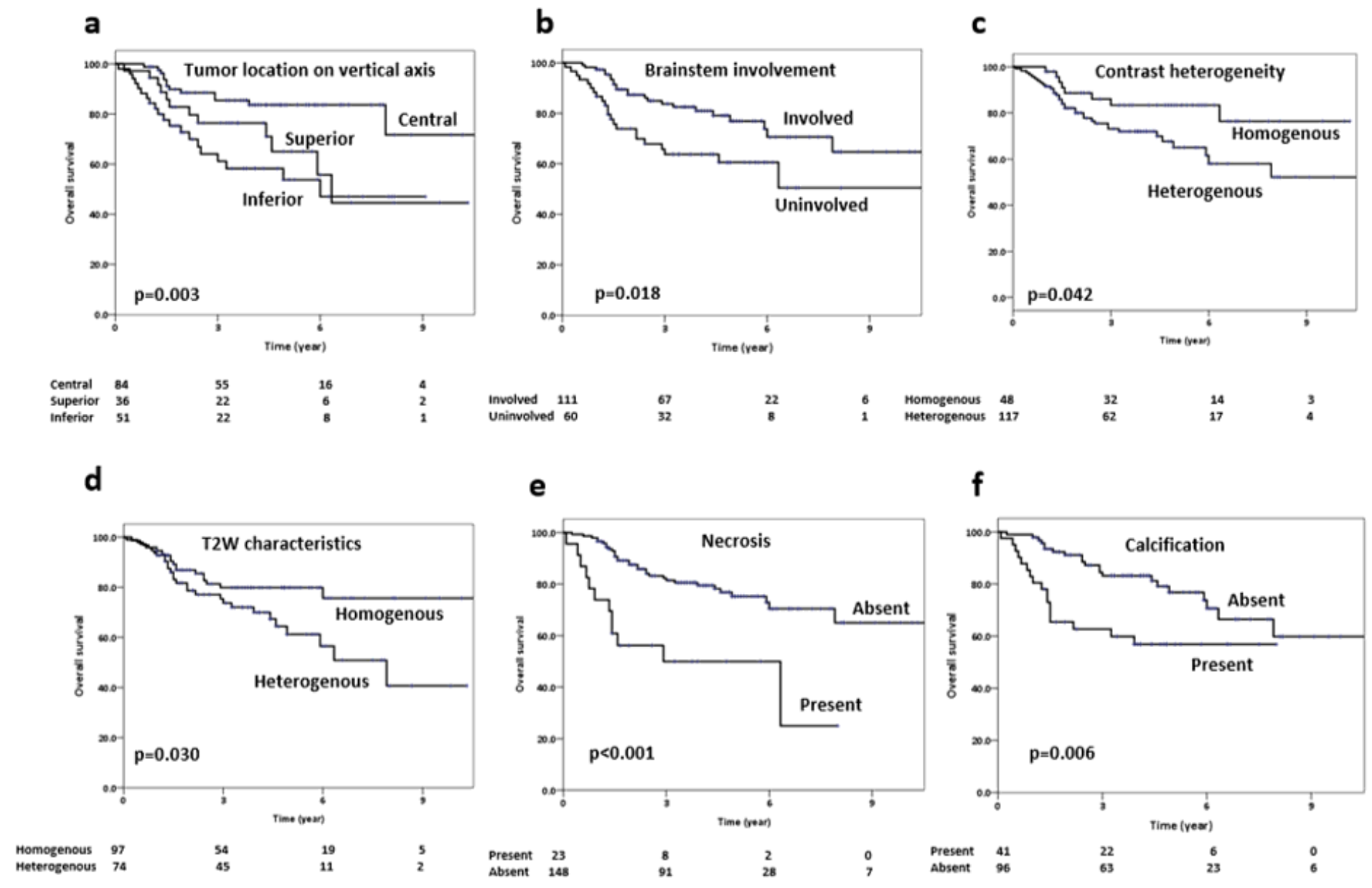

\section{Figure 1}

Kaplan Meier curves demonstrating the prognostic impact of semantic MRI features - tumor location on vertical axis (a), brainstem involvement (b), pattern of contrast-enhancement (c), T2-weighted characteristics (d), necrosis (e), and calcification ( $f$ ) on overall survival in medulloblastoma

\section{Supplementary Files}

This is a list of supplementary files associated with this preprint. Click to download.

- SupplementaryfileS1.docx

- SupplementaryfileS2.docx

- SupplementaryfileS3.tif

- SupplementaryfileS4.tif

- SupplementaryfileS5.tif 